\title{
Evolution of electron states at a narrow-gap semiconductor surface in an accumulation-layer formation process
}

\author{
Shuma Abe, ${ }^{*}$ Takeshi Inaoka, ${ }^{\dagger}$ and Masayuki Hasegawa \\ Department of Materials Science and Technology, Faculty of Engineering, Iwate University, 4-3-5 Ueda, Morioka, Iwate 020-8551, Japan
}

(Received 26 April 2002; revised manuscript received 6 September 2002; published 8 November 2002)

\begin{abstract}
Adsorption on a doped semiconductor surface often induces a gradual formation of a carrier-accumulation layer at the surface. Taking full account of a nonparabolic (NP) conduction-band dispersion of a narrow-gap semiconductor, such as InAs and InSb, we investigate the evolution of electron states at the surface in an accumulation-layer formation process. The NP conduction band is incorporated into a local-density-functional formalism. We compare the calculated results for the NP dispersion with those for the parabolic (P) dispersion with the band-edge effective mass. With increase in the accumulated carrier density $N_{S}$, the accumulated carriers for the NP conduction band start to be more localized in closer vicinity to the surface than those for the $\mathrm{P}$ one. As the bottoms of a few lowest subbands drop below the Fermi level one after another with increase in $N_{S}$, the nonparabolicity begins to have a great influence on the dispersion and the bottom of each of these subbands, particularly on those of the lowest subband. The present work provides a numerical basis for making a quantitative examination of surface electronic excitations in the accumulation-layer formation process.
\end{abstract}

DOI: 10.1103/PhysRevB.66.205309

PACS number(s): 73.20.At, 73.25.+i, 71.20.Nr

\section{INTRODUCTION}

Adsorption on a doped semiconductor surface often induces a gradual formation of a carrier-accumulation or carrier-depletion layer at the surface. Among these examples, it is known that a carrier-accumulation layer is readily formed on InAs surfaces. A clean InAs (110) surface suffers the same relaxation as a clean GaAs (110) surface. ${ }^{1-9}$ On a relaxed clean (110) surface of InAs, the In and As atoms in the top layer are displaced below and above the surface plane, respectively. This surface relaxation pushes an Inderived dangling-bond state above the conduction-band bottom and an As-derived one below the valence-band top. Accordingly, the band is flat at the clean well-defined InAs (110) surface. However, a tiny quantity of adsorbate, such as $\mathrm{H}$ atoms,${ }^{10} \mathrm{~N}$ atoms, ${ }^{11} \mathrm{O}_{2}$ molecules,,${ }^{12,13} \mathrm{Cl}_{2}$ molecules, ${ }^{13} \mathrm{Sb}$ atoms, ${ }^{14}$ and alkali-metal atoms, ${ }^{15,16}$ or a minute amount of surface defects ${ }^{17}$ induces a carrier-accumulation layer at an $n$-type InAs (110) surface. On the other band, there is already an accumulation layer at a clean (001) surface of $n$-type InAs prepared by the molecular-beam epitaxy (MBE) ${ }^{18-20}$ To prevent contamination and damage on the surface, Noguchi, Hirakawa, and Ikoma ${ }^{20}$ investigated a pristine surface immediately after MBE growth without exposure to air, and Bell, McConville, and Jones ${ }^{19}$ used a protective-overlayer technique. In the light of these careful preparations, the abovestated accumulation layer is considered to be intrinsic to the clean (001) surface. In addition, the accumulated carrier density depends upon whether the surface is As-stabilized or In-stabilized. ${ }^{20}$ At light doping, the accumulation layer becomes distinctly localized near the surface, while, at heavy doping, the accumulation only means that the carrier density in the semi-infinite system becomes enhanced in the neighborhood of the surface. With further increase in doping level, there occurs no substantial accumulation at the surface. ${ }^{21}$

There are several schemes available for calculating the carrier-density distribution and the effective one-electron po- tential self-consistently in the absence and the presence of a surface space-charge layer. To perform self-consistent calculations with reasonable effort, we often replace the surfacestate charges due to carrier transfer to or from the conduction band by a uniform charge distribution at the surface. The first one of these schemes is a Hartree calculation using a parameterized Morse potential for the effective potential. ${ }^{22,23}$ This parameterized scheme saves considerable effort in selfconsistent calculations of semi-infinite systems. The second one is a complete Hartree calculation of a carrier system in a semiconductor slab. ${ }^{10,24-26}$ The effective potential is calculated numerically without parametrizing it. The slab geometry makes it easier to obtain a self-consistent solution than the semi-infinite geometry. The third one is a local-densityapproximation (LDA) calculation of a carrier system in the semiconductor slab. ${ }^{27}$ The slab is taken to be sufficiently thick that the carrier-density distribution at the slab surface becomes equivalent to that at the surface of the semi-infinite system.

The presence of the accumulation layer has a remarkable influence on surface excitations of carriers often coupled with surface polar phonons. These surface excitations can be observed by high-resolution electron energy-loss spectroscopy (HREELS). As mentioned above, hydrogen adsorption on an $n$-type InAs (110) surface causes a gradual formation of the accumulation layer at the surface. For lightly doped InAs, Chen, Hermanson, and Lapeyre have clearly observed the evolution of the coupled surface-excitation modes in this formation process. ${ }^{10}$ The variation in the EEL spectrum can be explained by growth of quasi-two-dimensional plasmons coupled with surface polar phonons. On the other hand, there are some other EELS measurements on an intrinsic accumulation layer at a clean $n$-type InAs (001) surface prepared carefully. ${ }^{19,20}$ At light doping, quasi-two-dimensional plasmons occur in a sharply localized accumulation layer, ${ }^{20}$ while, at heavy doping, electronic excitations in the accumulation layer are virtually surface plasmons in a semi-infinite system with the carrier density enhanced near the surface. ${ }^{19}$ 
In relation to the EELS measurements, the surface excitations in the absence and the presence of a surface spacecharge layer have been calculated by means of a hydrodynamic theory combined with a step density-profile model, ${ }^{19,28,29}$ a semiclassical local-response theory formulated in Ref. 30, ${ }^{10,20,25}$ the random-phase approximation, ${ }^{31-34}$ and the time-dependent LDA. ${ }^{35}$ The last two accurate schemes taking account of nonlocal effects require the numerical results of thermal-equilibrium or ground states obtained from the Hartree and the LDA calculations, respectively.

The conduction band of a III-V semiconductor GaAs, InP, InAs, or InSb is appreciably or highly nonparabolic (NP), though almost isotropic. Particularly, a narrow-gap semiconductor InAs or InSb possesses a conspicuously NP conduction band. When this semiconductor is $n$-doped, the nonparabolicity has a great influence on electronic excitations in the conduction band. As for plasmons in the bulk, often coupled with polar phonons, there have been some theoretical schemes to incorporate the effects of the nonparabolicity into the RPA, in connection with Raman and infraredreflection measurements. The most advanced one among these schemes is to employ a NP dispersion as such obtained by a k·p method ${ }^{36}$ in the susceptibility of carriers. ${ }^{37,38} \mathrm{~A}$ simplified version of the $\mathbf{k} \cdot \mathbf{p}$ method with the spin-orbit splitting neglected is often used to facilitate the calculation of the susceptibility. ${ }^{37}$ However, a complete version of the $\mathbf{k} \cdot \mathbf{p}$ method including the spin-orbit splitting gives better and excellent agreement with experimental results of infrared-reflection measurements. ${ }^{38}$ The important point is to treat the dispersion around the Fermi level as accurately as possible, because plasmons are constituted of electronic transitions around the Fermi level.

This kind of accurate treatment of the NP dispersion is not so advanced in calculations of surface states and excitations as in those of bulk states and excitations. One simple scheme for surface excitations is to employ a parabolic (P) dispersion with its effective mass modified. ${ }^{33-35,39}$ The effective mass is so adjusted that the surface-plasmon energy at surfaceparallel wave number $Q=0$ accords with that for the NP band obtained by the complete version of the $\mathbf{k} \cdot \mathbf{p}$ method. However, this scheme can give a good description of surface plasmons only in a small $Q$ range. In another scheme treating an accumulation layer, the EEL spectrum has been calculated by using a step profile of the carrier density that approximates to the accurate density profile in thermal equilibrium taking account of the nonparabolicity. ${ }^{28,29}$ The latter density profile is calculated by the modified Thomas-Fermi approximation that involves the NP band obtained by the simplified version of the $\mathbf{k} \cdot \mathbf{p}$ method. ${ }^{40}$ However, this is a simplified scheme to calculate the EEL spectrum with reasonable effort. It is anticipated that the NP dispersion is incorporated in accurate schemes, such as the RPA and the time-dependent LDA, in a complete manner.

In the present work, we investigate the evolution of electron states at a narrow-gap semiconductor surface in an accumulation-layer formation process. We incorporate a NP conduction-band dispersion obtained by the complete version of the $\mathbf{k} \cdot \mathbf{p}$ method into the LDA formalism. We build up an exchange potential at finite temperatures that takes full account of the NP dispersion. Our calculation elucidates the carrier-density distribution, the effective one-electron potential, and the constituent subbands at each stage of the formation process. We evaluate the effects of the nonparabolicity by comparing the results for the NP dispersion with those for the $\mathrm{P}$ dispersion with the band-edge effective mass. It turns out that, with increase in the accumulated carrier density, the nonparabolicity begins to exercise a great influence on the dispersion of a few lowest subbands around the Fermi level. This implies that the nonparabolicity profoundly affects the electronic excitations in the accumulation layer. The present work furnishes a numerical basis for the next quantitative study of surface electronic excitations in the accumulationlayer formation process.

\section{THEORY}

In this section, we describe a theoretical framework for our following analysis. For convenience of calculation, we employ a doped semiconductor slab thick enough to replace a semi-infinite system even in the presence of heavy carrier accumulation at the surface. Our carrier system is assumed to be in a uniform background which is electrically positive due to ionized donors and whose polarization is described by a static dielectric constant $\varepsilon_{0}$. In $n$-type narrow-gap semiconductors, such as $n$-InAs and $n$-InSb, the combination of an extremely small effective mass and a large dielectric constant gives a very large effective Bohr radius. Therefore, increasing the doping level readily leads to such a high effective density of carriers that an impurity band due to donors merges into the conduction band. In this case, ionized donors can be spread out into a uniform distribution of positive charges. The characteristic length to describe the penetration of electron states into the vacuum $(\sim 1 \AA)$ is much shorter than that to describe the carrier-density variation in an accumulation layer (several tens of $\AA$ ). Accordingly, it is a good approximation to impose a boundary condition that wave functions of carriers vanish at the background surface. If surface states release some of their electrons into the conduction band, the surface becomes positively charged, and a carrier-accumulation layer is formed just on the inside of the positively charged surface. For simplicity of calculation, we describe the charged surface by a uniform distribution of surface charges that extends right outside and along each background surface..$^{10,22-29,31,32,35}$ We assume the charge neutrality of the whole system that is composed of the carrier system, the positive background, and the surface-charge distribution.

We take a $z$-axis to be normal to the surfaces of the background slab with thickness $2 l$. The surface planes are located at $z=0$ and $2 l$. We employ the following Schrödinger equation that can be applied to a general energy dispersion of the conduction band:

$$
\left[E_{C}(-i \nabla)+V(z)\right] \Psi(\mathbf{R}, z)=E \Psi(\mathbf{R}, z),
$$

where $E_{C}(-i \nabla)$ is the conduction-band dispersion $E_{C}(\mathbf{k})$ with its wave vector $\mathbf{k}$ replaced by a differential operator $-i \nabla$. The symbols $\mathbf{R}$ and $V(z)$ denote a surface-parallel 
position vector and an effective one-electron potential, respectively. The above equation with $E_{C}(-i \nabla)$ is derived by the Wannier representation, and $\Psi(\mathbf{R}, z)$ represents an envelope function in terms of Wannier functions. ${ }^{41}$ This equation is valid, when the potential $V(z)$ varies slowly on the scale of lattice constants. Each eigenfunction for surface-parallel wave vector $\mathbf{K}$ and subband $j$ can be expressed by a product of the surface-parallel component and the surface-normal one as

$$
\Psi_{\mathbf{K}, j}(\mathbf{R}, z)=\frac{1}{\sqrt{A}} \exp (i \mathbf{K} \cdot \mathbf{R}) \phi_{\mathbf{K}, j}(z),
$$

where $A$ denotes the area of the background slab. Under the boundary condition mentioned above, the surface-normal component $\phi_{\mathbf{K}, j}(z)$ can be expanded in a Fourier sine series as

$$
\phi_{\mathbf{K}, j}(z)=\sum_{\nu=1}^{\infty} a_{\nu}^{(\mathbf{K}, j)} \frac{1}{\sqrt{l}} \sin \left(\frac{\nu \pi}{2 l} z\right) .
$$

By this expansion, the differential equation Eq. (1) can be transformed into a matrix-eigenvalue problem of the form,

$$
\sum_{\nu=1}^{\infty} M_{\lambda \nu} a_{\nu}^{(K, j)}=E(K, j) a_{\lambda}^{(K, j)}
$$

where the matrix element $M_{\lambda \nu}$ is written as

$$
M_{\lambda \nu}=E_{C}\left(k_{\lambda}\right) \delta_{\lambda \nu}+\frac{1}{l} \int_{0}^{2 l} d z V(z) \sin \left(\frac{\lambda \pi}{2 l} z\right) \sin \left(\frac{\nu \pi}{2 l} z\right) .
$$

In Eqs. (4) and (5), $E_{C}(\mathbf{k}), E(\mathbf{K}, j)$, and $a_{\lambda}^{(\mathbf{K}, j)}$ are expressed merely as $E_{C}(k)(k=|\mathbf{k}|), E(K, j)$, and $a_{\lambda}^{(K, j)}(K=|\mathbf{K}|)$, respectively, because these quantities are isotropic with respect to $\mathbf{k}$ or $\mathbf{K}$. The wave number $k_{\lambda}$ is defined by

$$
k_{\lambda}=\sqrt{\mathbf{K}^{2}+(\lambda \pi / 2 l)^{2}},
$$

and $\delta_{\lambda \nu}$ signifies the Kronecker delta.

We can calculate the conduction-band dispersion by using the complete version of the k•p method in Ref. 36. The energy $E_{C}$ for each $k$ can be obtained by taking the largest solution of an algebraic equation for $E^{\prime}$,

$$
E^{\prime}\left(E^{\prime}+E_{G}\right)\left(E^{\prime}+E_{G}+\Delta_{0}\right)-k^{2} P^{2}\left(E^{\prime}+E_{G}+2 \Delta_{0} / 3\right)=0,
$$

where $E_{G}$ and $\Delta_{0}$ denote the energy gap and the spin-orbit splitting, respectively. The solution of $E^{\prime}$ is related to $E_{C}(k)$ by $E^{\prime}=E_{C}(k)-\hbar^{2} k^{2} / 2 m_{0}$ with the free-electron mass $m_{0}$. The coefficient $P$ is given by

$$
P^{2}=\frac{3 \hbar^{2}\left(1 / m_{0}^{*}-1 / m_{0}\right)}{2\left[2 / E_{G}+1 /\left(E_{G}+\Delta_{0}\right)\right]},
$$

with the band-edge effective mass $m_{0}^{*}$.

The carrier-density distribution $n(z)$ and the effective one-electron potential $V(z)$ can be obtained by solving Eqs. (4) and (5) and the following equations self-consistently:

$$
\begin{gathered}
n(z)=\frac{1}{\pi} \sum_{j} \int_{0}^{\infty} d K \frac{K\left|\phi_{K, j}(z)\right|^{2}}{\exp [\beta\{E(K, j)-\mu\}]+1}, \\
N_{S}=\frac{1}{2} \int_{0}^{2 l}\left[n(z)-n_{0}\right] d z, \\
V(z)=V_{H}[n ; z]+V_{X}[n] .
\end{gathered}
$$

Here, the symbol $\beta$ is defined by $\beta=1 / k_{B} T$ in terms of the Boltzmann constant $k_{B}$ and the absolute temperature $T$, and $n_{0}$ is the donor density or the carrier density in the bulk. The density $N_{S}$ designates the accumulated carrier number per unit area for each surface. Equations (9) and (10) are employed to determine the Fermi level $\mu$ for given $n_{0}$ and $N_{S}$. The effective potential $V$ is made up of the Hartree potential $V_{H}$ and the exchange potential $V_{X}$. By solving Poisson's equation,

$$
\frac{d^{2}}{d z^{2}} V_{H}[n ; z]=-\frac{4 \pi e^{2}}{\varepsilon_{0}}\left[n(z)-n_{0}\right]
$$

we can obtain the following form of $V_{H}$ :

$$
V_{H}[n ; z]=-\frac{4 \pi e^{2}}{\varepsilon_{0}} \int_{z}^{l} d z^{\prime}\left(z^{\prime}-z\right)\left[n\left(z^{\prime}\right)-n_{0}\right] .
$$

We can take account of the exchange-correlation (XC) effects by means of the LDA. However, as far as we know, there is no parameterized XC potential available for a NP conduction-band dispersion. In the present work, we consider only the exchange $(X)$ potential $V_{X}$ that can be calculated with reasonable effort. The $X$ part of the Helmholtz free energy for a bulk electron system can be expressed as ${ }^{42}$

$$
\begin{aligned}
F_{X} & =-V_{0} \int \frac{d^{3} \mathbf{k}}{(2 \pi)^{3}} \int \frac{d^{3} \mathbf{q}}{(2 \pi)^{3}} \frac{4 \pi e^{2}}{\varepsilon_{0}|\mathbf{k}-\mathbf{q}|^{2}} f(k) f(q) \\
& =-V_{0} \frac{e^{2}}{2 \pi^{3} \varepsilon_{0}} \int_{0}^{\infty} d k \int_{0}^{\infty} d q k q f(k) f(q) \ln \left|\frac{k+q}{k-q}\right|
\end{aligned}
$$

where a symbol $V_{0}$ and a multiplied factor $4 \pi e^{2} / \varepsilon_{0}|\mathbf{k}-\mathbf{q}|^{2}$ are a system volume and a Fourier transform of the Coulomb potential, respectively. The Fermi-Dirac distribution function $f(k)$ in Eq. (14) is defined by

$$
f(k)=\frac{1}{\exp \left[\beta E_{C}(k)-\eta_{0}\right]+1},
$$

with $\eta_{0}=\beta \mu$. On the other band, the electron number $N$ is written as

$$
N=2 V_{0} \int \frac{d^{3} \mathbf{k}}{(2 \pi)^{3}} f(k)=\frac{V_{0}}{\pi^{2}} \int_{0}^{\infty} d k k^{2} f(k) .
$$

This equation is used to determine $\eta_{0}$ or $\mu$ for given electron density $n_{0}=N / V_{0}$ and temperature $T$. The $X$ potential $V_{X}$ is given by 


$$
V_{X}=\left(\frac{\partial F_{X}}{\partial N}\right)_{T, V_{0}}=\frac{\left(\partial F_{X} / \partial \eta_{0}\right)_{T, V_{0}}}{\left(\partial N / \partial \eta_{0}\right)_{T, V_{0}}},
$$

where the numerator and the denominator are expressed as

$$
\begin{aligned}
\left(\frac{\partial F_{X}}{\partial \eta_{0}}\right)_{T, V_{0}}= & -V_{0} \frac{e^{2}}{2 \pi^{3} \varepsilon_{0}} \int_{0}^{\infty} d k \int_{0}^{\infty} d q k q f(k) f(q) \\
& \times\{2-f(k)-f(q)\} \ln \left|\frac{k+q}{k-q}\right|,
\end{aligned}
$$

and

$$
\left(\frac{\partial N}{\partial \eta_{0}}\right)_{T, V_{0}}=\frac{V_{0}}{\pi^{2}} \int_{0}^{\infty} d k k^{2} f(k)\{1-f(k)\}
$$

respectively. The potential $V_{X}$ in Eq. (17) is used in our self-consistent calculation for $n(z)$ and $V(z)$. For a $\mathrm{P}$ conduction band, there is a parametrized form of $V_{X}$ as follows: ${ }^{43}$

$$
V_{X}=-\left(\frac{9}{4 \pi^{2}}\right)^{1 / 3} \frac{e^{2}}{\varepsilon_{0} r_{s}^{*} a_{B}^{*}} G\left(\frac{k_{B} T}{\varepsilon_{F}}\right),
$$

where the effective Bohr radius $a_{B}^{*}$ is defined by $a_{B}^{*}$ $=\varepsilon_{0} \hbar^{2} / m_{0}^{*} e^{2}$. The density parameter $r_{s}^{*}$ is related to the carrier density $n$ by $(4 \pi / 3)\left(r_{s}^{*} a_{B}^{*}\right)^{3} n=1$. The temperature factor $G\left(k_{B} T / \varepsilon_{F}\right)$, where $\varepsilon_{F}$ stands for the Fermi energy defined at $T=0$, is given by

$$
G(x)=\frac{1+c_{1} x^{2}+c_{2} x^{4}+c_{3} x^{6}}{1+\left(c_{1}+\pi^{2} / 12\right) x^{2}+c_{4} x^{4}+c_{3} x^{5}\left(\sqrt{x / 2 \pi}+3 x^{2} / 2\right)},
$$

with $c_{1}=41.775, c_{2}=27.390, c_{3}=4287.2$, and $c_{4}=50.605$. We employ a standard iteration scheme to obtain a selfconsistent solution of Eqs. (4), (5), and (9)-(11).

We can decompose $n(z)$ into contributions of the constituent subbands. The component $n_{j}(z)$, namely, the contribution of subband $j$ to $n(z)$ is given by the summand on the r.h.s. of Eq. (9). By integrating $n_{j}(z)$ in $z$, we can obtain the areal carrier density of subband $j$ as

$$
N_{j}=\frac{1}{\pi} \int_{0}^{\infty} d K \frac{K}{\exp [\beta\{E(K, j)-\mu\}]+1} .
$$

\section{RESULTS AND DISCUSSION}

By means of the theoretical framework in Sec. II, we investigate the evolution of electron states at a narrow-gap semiconductor surface in an accumulation-layer formation process. As described in Sec. I, hydrogen adsorption on an $n$-type InAs (110) surface induces a gradual formation of a carrier-accumulation layer at the surface. Our calculations are concerned with this case. The donor density $n_{0}$ and the temperature $T$ are taken to be $n_{0}=1.3 \times 10^{16} \mathrm{~cm}^{-3}$ and $T$ $=300 \mathrm{~K}$, respectively, corresponding to the HREELS measurement in Ref. 10. We employ the value of the static di-

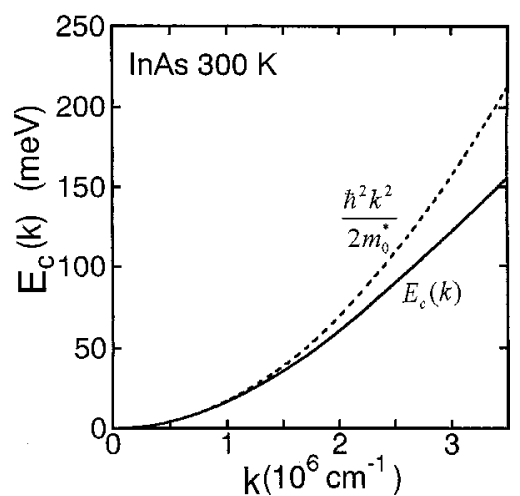

FIG. 1. Energy dispersion $E_{C}(k)$ of the conduction band of InAs at room temperature, in comparison to the parabolic dispersion with the band-edge effective mass $m_{0}^{*}$.

electric constant $\varepsilon_{0}=15.15,{ }^{44}$ and our background slab is $600 \mathrm{~nm}$ thick.

Before examining electron states at the surface, we analyze the energy dispersion of the conduction band in the bulk. Figure 1 shows the dispersion $E_{C}(k)$ in comparison to the parabolic dispersion with the band-edge effective mass $m_{0}^{*}$. The dispersion $E_{C}(k)$ has been calculated by using the band gap $E_{G}=0.3543 \mathrm{eV},{ }^{45}$ the spin-orbit splitting $\Delta_{0}$ $=0.381 \mathrm{eV},{ }^{46}$ and $m_{0}^{*}=0.02195 m_{0} \cdot{ }^{47}$ The band gap $E_{G}$ depends upon $T$ remarkably, and the above $E_{G}$ value is for $T$ $=295 \mathrm{~K}$. With increase in $k$, the dispersion $E_{C}(k)$ begins to deviate downward from the parabolic dispersion conspicuously, and changes into a linearlike increase. This noticeable deviation implies a high nonparabolicity. These two dispersions are employed in our calculations of electron states at the surface.

Hereafter, we investigate the evolution of electron states at the surface in an accumulation-layer formation process. Figures 2(a) and 2(b) exhibit the variation of the carrierdensity distribution $n(z)$ and the effective one-electron potential $V(z)$ in the formation process, respectively. The accumulated carrier density $N_{S}$ is varied from 0 to 1.6 in units of $10^{12} \mathrm{~cm}^{-2}$. Each $N_{S}$ value is assigned to a pair of a full curve and a broken one. The full curves and the broken ones are obtained by using the NP dispersion $E_{C}(k)$ and the P one with $m_{0}^{*}$ in Fig. 1, respectively. With increase in $N_{S}$, a peak appears and grows in the carrier-density distribution $n(z)$. This indicates a gradual formation of an accumulation layer. The carrier density $n(z)$ approaches its bulk value $n_{0}(=1.3$ $\times 10^{16} \mathrm{~cm}^{-3}$ ) well below the surface. While $N_{S}$ is small, there is only a small difference in $n(z)$ between the NP and P dispersions. With increase in $N_{S}$, however, the peak for the NP dispersion begins to become higher and closer to the surface than that for the $\mathrm{P}$ dispersion. This implies that the accumulated carriers are more localized in a nearer neighborhood of the surface in the NP dispersion. The variation of $V(z)$ corresponds to that of $n(z)$. The value of $V(z)$ is measured from its value well inside the material. At smaller $N_{S}$ values, there exists only a small difference in $V(z)$ between the NP and P dispersions. With increase in $N_{S}$, however, the potential curve for the NP dispersion begins to deviate from that for the $\mathrm{P}$ dispersion to the surface. The peak in $n(z)$ 

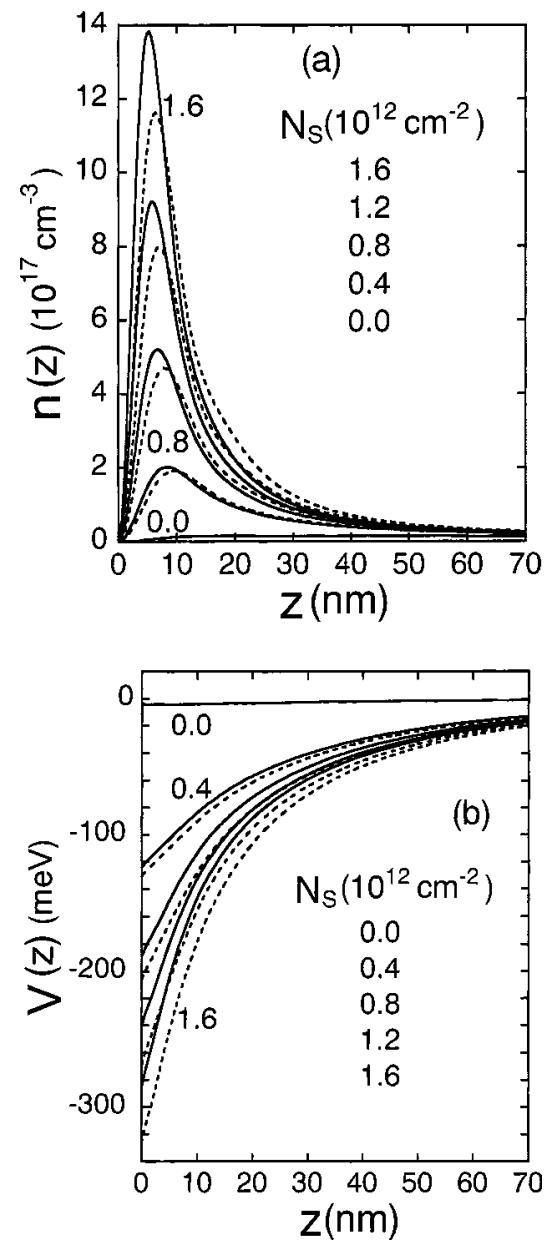

FIG. 2. Evolution of (a) the carrier-density distribution $n(z)$ and (b) the effective one-electron potential $V(z)$ in the accumulationlayer formation process. Each pair of a full curve and a broken one corresponds to a value of the accumulated carrier density $N_{S}$, and the full curves and the broken ones are for the nonparabolic conduction band and the parabolic one, respectively.

arises from a screening effect of the carriers on the positively charged surface. As displayed in Fig. 1, the NP dispersion curve departs downward from the $\mathrm{P}$ dispersion curve. This leads to a shorter screening length in the NP dispersion, and consequently a more localization of the carriers in closer proximity to the surface. This situation can be understood within the P-dispersion framework as well, namely, by recalling that the $\mathrm{P}$ dispersion with a larger effective mass, whose curve lies lower in energy, results in a shorter Thomas-Fermi screening length.

Next, we examine subbands that constitute the carrieraccumulation layer. Figure 3 shows the energy dispersion $E(K, j)$ of the subbands $j$ at $N_{S}=0.1 \times 10^{12} \mathrm{~cm}^{-2}$ (a), 0.8 $\times 10^{12} \mathrm{~cm}^{-2}$ (b), and $1.6 \times 10^{12} \mathrm{~cm}^{-2}$ (c). The capital $K$ on the abscissa denotes the surface-parallel wave number. The subbands are numbered by $j(=1,2,3, \ldots)$ in order of increasing energy. Full curves and broken ones exhibit the results for the NP and P conduction bands, respectively. The horizontal lines $\mu_{\mathrm{np}}$ and $\mu_{\mathrm{p}}$ display the Fermi levels for the NP and $\mathrm{P}$ conduction bands, respectively. Figure 4 shows the decomposition of the carrier-density distribution $n(z)$ into
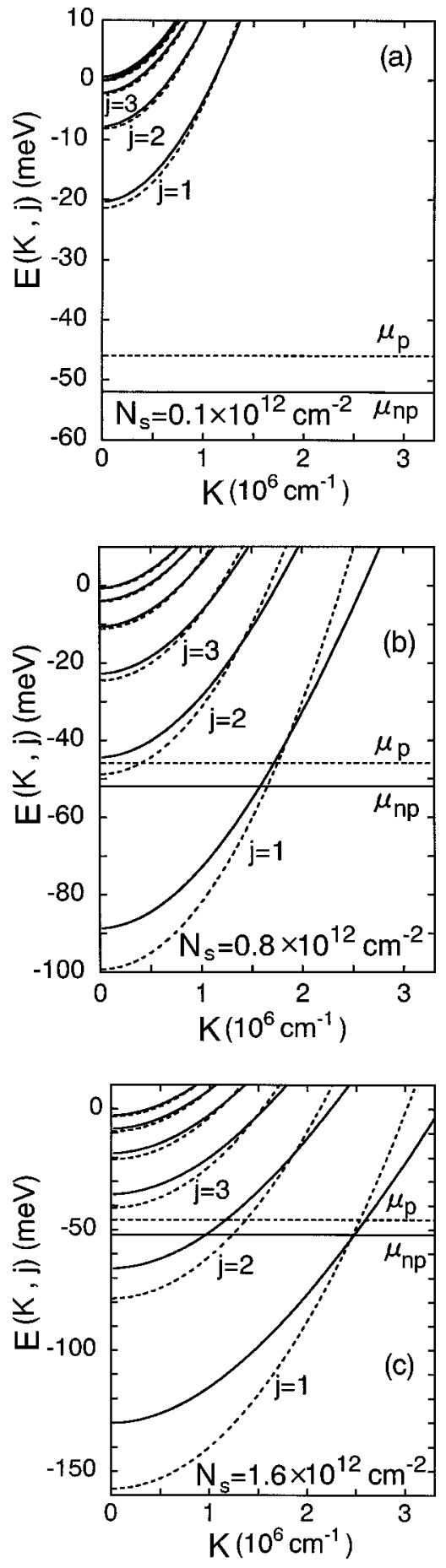

FIG. 3. Energy dispersion $E(K, j)$ of the subbands $j$ at $N_{S}$ $=0.1 \times 10^{12} \mathrm{~cm}^{-2}(\mathrm{a}), 0.8 \times 10^{12} \mathrm{~cm}^{-2}(\mathrm{~b})$, and $1.6 \times 10^{12} \mathrm{~cm}^{-2}(\mathrm{c})$. The full curve and the broken one for each subband $j$ represent the dispersion for the nonparabolic conduction band and that for the parabolic one, respectively. The horizontal lines $\mu_{\mathrm{np}}$ and $\mu_{\mathrm{p}}$ locate the Fermi levels for the nonparabolic and parabolic conduction bands, respectively.

contributions of the constituent subbands at $N_{S}=0.1$ $\times 10^{12} \mathrm{~cm}^{-2}$ (a), $0.8 \times 10^{12} \mathrm{~cm}^{-2}$ (b), and $1.6 \times 10^{12} \mathrm{~cm}^{-2}$ (c). The panels (a)-(c) in this figure correspond to those in Fig. 3, respectively. The density $n_{j}$ signifies the component 

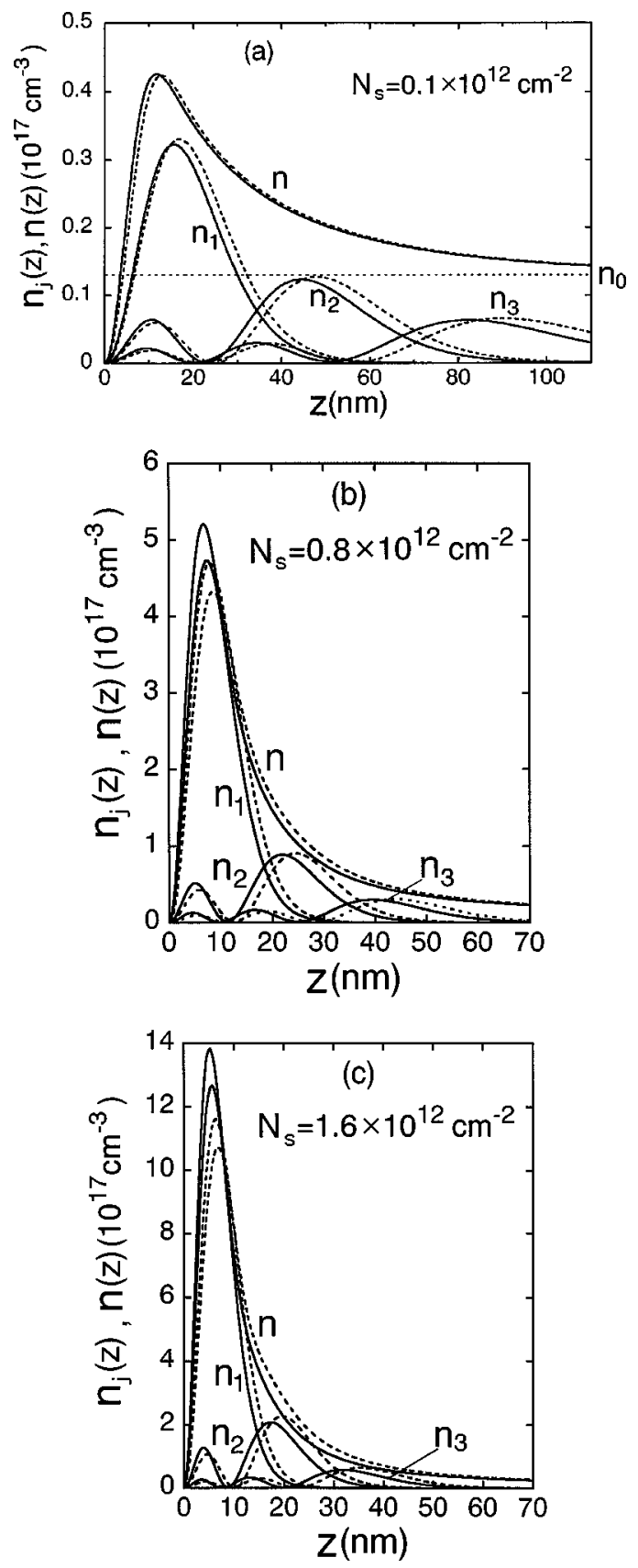

FIG. 4. Decomposition of the carrier-density distribution $n(z)$ into contributions $n_{j}(z)$ of the constituent subbands $j$ at $N_{S}=0.1$ $\times 10^{12} \mathrm{~cm}^{-2}$ (a), $0.8 \times 10^{12} \mathrm{~cm}^{-2}$ (b), and $1.6 \times 10^{12} \mathrm{~cm}^{-2}$ (c). The full curve and the broken one for $n$ and each $n_{j}$ indicate the density for the nonparabolic conduction band and that for the parabolic one, respectively. The horizontal dotted line in (a) designates the bulk carrier density $n_{0}$.

of subband $j$. As in Fig. 3, full curves and broken ones designate the results for the NP and P conduction bands, respectively. A dotted horizontal line in Fig. 4(a) indicates the bulk carrier density $n_{0}$. When $N_{S}$ is small [see Figs. 3(a) and 4(a)], even the bottom of the lowest subband is considerably above the Fermi level $\mu$, and the constituent subbands accommodate a small number of thermally excited carriers. These subbands make a comparable contribution to $n(z)$ with a small peak. There is a small difference in $E(K, j)$ and $n_{j}(z)$ between the NP and P conduction bands. With an increase in $N_{S}$ to $0.8 \times 10^{12} \mathrm{~cm}^{-2}$, the bottom of the lowest subband drops significantly below $\mu$, and the bottom of the second lowest subband becomes close to $\mu$ [see Fig. 3(b)]. The dispersion of the lowest subband for the NP conduction band departs conspicuously from that for the $\mathrm{P}$ conduction band. The lowest subband with its bottom below $\mu$ constitutes the main part of the grown peak in $n(z)$, and the subbands with $j=2$ and 3 form a tail part in $n(z)$ extending into a deeper region [see Fig. 4(b)]. With a further increase in $N_{S}$ to $1.6 \times 10^{12} \mathrm{~cm}^{-2}$, the lowest subband sinks further in energy, and its dispersion for the NP conduction band departs greatly from that for the P conduction band [see Fig. 3(c)]. Simultaneously, the bottom of the second lowest subband also falls below $\mu$, and there appears a noticeable departure in dispersion in this subband as well. By comparing Figs. 4(a)-4(c), we can follow the variation in the carrier-density distribution of each subband. The lowest subband of $j=1$ has a one-peak profile, and the density component $n_{j}(z)$ of $j>1$ shows an oscillation with $j-1$ nodes. As the positive surface-charge density due to surface states becomes larger with increase in $N_{S}$, the carriers in each subband begin to be attracted to the surface, and the peak in $n(z)$ becomes sharper and higher. With an increase in $N_{S}$, it becomes more evident that the density curve of the lowest subband for the NP conduction band deviates from that for the $\mathrm{P}$ conduction band to the surface with its peak growth, which results in a more localization of the accumulated carriers in closer vicinity to the surface.

Figure 5(a) exhibits the $N_{S}$ dependence of the subband bottoms $\varepsilon_{j}$. The horizontal lines $\mu_{\mathrm{np}}$ and $\mu_{\mathrm{p}}$ indicate the Fermi levels for the NP and P conduction bands, respectively. A vertical arrow labeled $k_{B} T_{R}$ shows an energy separation corresponding to room temperature $T=300 \mathrm{~K}$. Figure 5 (b) displays the $N_{S}$ dependence of the areal carrier density $N_{j}$ of subband $j$. The density $N_{j}$ can be obtained by integrating $n_{j}(z)$ in $z$. In Fig. 5(a) or 5(b), filled circles connected by full lines and open circles combined with broken lines represent the results for the NP and P conduction bands, respectively. To understand the behavior in Fig. 5, we pay special attention to the lowest subband in Figs. 3(b) and 3(c). Though there is a great difference in dispersion between the NP and P conduction bands, the two dispersion curves of this subband must cross the corresponding Fermi levels nearly at the same $K$ value to satisfy the accumulated carrier density $N_{S}$ specified. This explains why the $N_{1}$ value for the NP conduction band differs only slightly from that for the $\mathrm{P}$ conduction band, as shown in Fig. 5(b). As is consistent with the conduction-band dispersions in Fig. 1, with an increase in $K$, the subband energy $E(K, j=1)$ for the NP conduction band rises at a slower rate than that for the $\mathrm{P}$ conduction band. Accordingly, the subband bottom for the NP conduction band must be higher than that for the P conduction band, in order that the two dispersion curves should intersect with the $\mu$ lines nearly at the same $K$ value. Figure 5(a) clarifies that, with an increase in $N_{S}$, the bottoms of a few lowest subbands drop below $\mu$ one after another, and the bottom for the NP conduction band start to deviate upward from those 

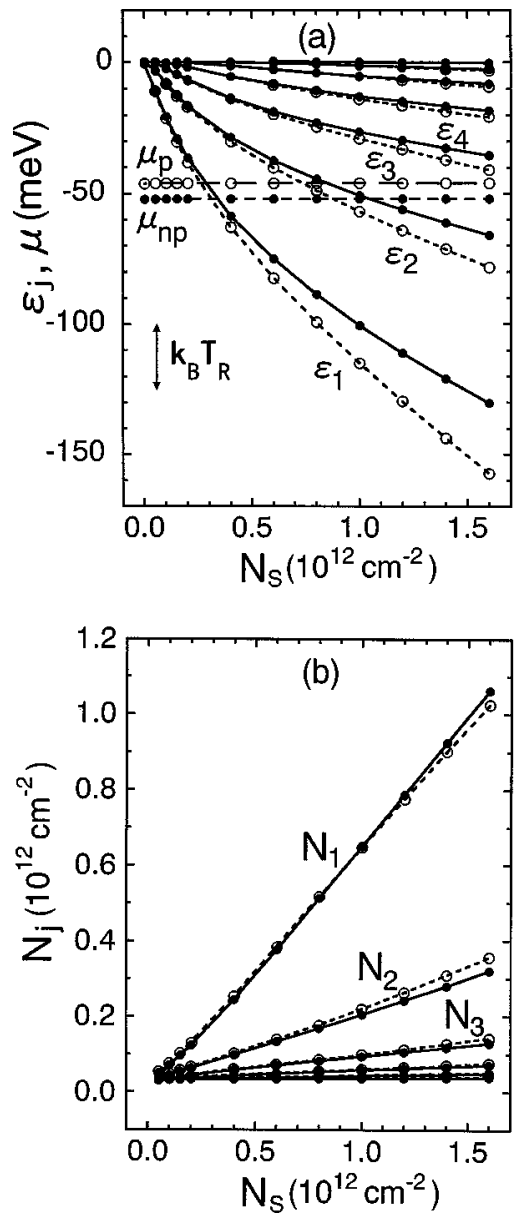

FIG. 5. $N_{S}$ dependence of (a) the subband bottoms $\varepsilon_{j}$ and (b) the areal carrier density $N_{j}$ of the constituent subbands $j$. In each panel, the filled circles connected by full lines and the open circles combined with broken lines exhibit the results for the nonparabolic conduction band and those for the parabolic one, respectively. In panel (a), the energy levels $\mu_{\mathrm{np}}$ and $\mu_{\mathrm{p}}$ display the Fermi level for the nonparabolic conduction band and that for the parabolic one, respectively. The vertical arrow marked $k_{B} T_{R}$ shows an energy separation corresponding to room temperature.

for the $\mathrm{P}$ conduction band in a conspicuous manner. As exhibited in Fig. 5(b), the density $N_{j}$ of each subband increases linearly with $N_{S}$. There can be no substantial difference in $N_{j}$ between the NP and P conduction bands, because the $N_{j}$ values must satisfy the given $N_{S}$ value.

\section{SUMMARY}

Taking full account of a nonparabolic conduction-band dispersion of an $n$-type narrow-gap semiconductor, we have analyzed the evolution of electron states at the surface in an accumulation-layer formation process. With increase in wave number $k$, the nonparabolic (NP) dispersion of the conduction band starts to depart downward from the parabolic (P) dispersion with the band-edge effective mass, and evolves into a linearlike increase. This NP dispersion has been incorporated in the local-density-functional formalism. We have calculated electron states at the surface, and have compared the results for the NP conduction band with those for the $\mathrm{P}$ one. The evolution of the electron states with increase in the depleted carrier density $N_{S}$ can be summarized as follows:

(1) With increase in $N_{S}$, the accumulated carriers for the NP conduction band begin to be more localized in closer vicinity to the surface than those for the $\mathrm{P}$ one. This can be ascribed to the fact that the carriers in the NP conduction band possess a shorter screening length than those in the $\mathrm{P}$ one.

(2) With increase in $N_{S}$, it becomes more obvious that the carrier-density curve of the lowest subband for the NP conduction band deviates from that for the $\mathrm{P}$ one to the surface with its peak growth. This is largely responsible for the above-mentioned localization of the accumulated carriers in the nearer neighborhood of the surface.

(3) As the bottoms of a few lowest subbands fall below the Fermi level one after another with increase in $N_{S}$, the nonparabolicity of the conduction band starts to exert a great and growing influence on the dispersion and the bottom of each of these subbands. This influence is greatest in the lowest subband. The subband bottom for the NP conduction band becomes remarkably higher than that for the $\mathrm{P}$ one, and with increase in the surfaceparallel wave number, the subband energy rises from the bottom more slowly in the NP conduction band than in the $\mathrm{P}$ one.

(4) The areal carrier density of each subband increases linearly with $N_{S}$. The nonparabolicity has no substantial influence on this density increase.

The present work forms a numerical basis for quantitative calculation of the evolution of plasmons often coupled with polar phonons in the accumulation-layer formation process. This evolution can be observed by HREELS. Among various possible intrasubband and intersubband excitations, intrasubband excitations in the lowest subband play a major role, ${ }^{26}$ and these excitations are composed of electronic transitions in the lowest subband around the Fermi level. As shown clearly in Sec. III, the nonparabolicity has the most pronounced effect on the energy dispersion of the lowest subband. This implies that accurate treatment of the nonparabolic subband dispersion, especially around the Fermi level, is indispensable for quantitative analysis of electronic excitations in the accumulation layer.

\section{ACKNOWLEDGMENTS}

This work is supported by a Grant-in-Aid for Scientific Research from the Ministry of Education, Science, Sport, and Culture under Grant No. 13640315. The numerical calculations in the present work were performed at the Iwate University Super Computing and Information Sciences Center and at the Information Synergy Center of Tohoku University. 
*Present address: DNP Jyouhou Shisutemu Co. Ltd., 1-12-18

Saien, Morioka, Iwate 020, Japan.

${ }^{\dagger}$ Author to whom correspondence should be addressed.

${ }^{1}$ C. B. Duke, S. L. Richardson, A. Paton, and A. Kahn, Surf. Sci. 127, L135 (1983).

${ }^{2}$ S. Y. Tong, W. N. Mei, and G. Xu, J. Vac. Sci. Technol. B 2, 393 (1984).

${ }^{3}$ L. Smith, T. E. Derry, and J. F. van der Veen, Surf. Sci. 150, 245 (1985).

${ }^{4}$ R. M. Feenstra, J. A. Stroscio, J. Tersoff, and A. P. Fein, Phys. Rev. Lett. 58, 1192 (1987).

${ }^{5}$ J. E. Gayone, R. G. Pregliasco, G. R. Gómez, E. A. Sánchez, and O. Grizzi, Phys. Rev. B 56, 4186 (1997).

${ }^{6}$ C. Mailhiot, C. B. Duke, and D. J. Chadi, Surf. Sci. 149, 366 (1985)

${ }^{7}$ A. C. Ferraz and G. P. Srivastava, Surf. Sci. 182, 161 (1987).

${ }^{8}$ G.-X. Qian, R. M. Martin, and D. J. Chadi, Phys. Rev. B 37, 1303 (1988).

${ }^{9}$ J. L. A. Alves, J. Hebenstreit, and M. Scheffler, Phys. Rev. B 44, 6188 (1991).

${ }^{10}$ Y. Chen, J. C. Hermanson, and G. J. Lapeyre, Phys. Rev. B 39, 12682 (1989).

${ }^{11}$ T. Van Gemmeren, S. Rossi Salmagne, and W. Mönch, Appl. Surf. Sci. 65/66, 625 (1993).

${ }^{12}$ H.-U. Baier, L. Koenders, and W. Mönch, Solid State Commun. 58, 327 (1986).

${ }^{13}$ K. Smit, L. Koenders, and W. Mönch, J. Vac. Sci. Technol. B 7, 888 (1989).

${ }^{14}$ C. Nowak, J. Krujatz, A. Märkl, C. Meyne, A. Chassé, W. Braun, W. Richter, and D. R. T. Zahn, Surf. Sci. 331-333, 619 (1995).

${ }^{15}$ V. Y. Aristov, G. Le Lay, Le Thanh Vinh, K. Hricovini, and J. E. Bonnet, Phys. Rev. B 47, 2138 (1993).

${ }^{16}$ V. Y. Aristov, G. Le Lay, P. Soukiassian, K. Hricovini, J. E. Bonnet, J. Osvald, and O. Olsson, Europhys. Lett. 26, 359 (1994).

${ }^{17}$ H. S. Karlsson, R. Viselga, and U. O. Karlsson, Surf. Sci. 402404, 590 (1998).

${ }^{18}$ L. Ö. Olsson, C. B. M. Andersson, M. C. Håkansson, J. Kanski, L. Ilver, and U. O. Karlsson, Phys. Rev. Lett. 76, 3626 (1996).

${ }^{19}$ G. R. Bell, C. F. McConville, and T. S. Jones, Phys. Rev. B 54, 2654 (1996).

${ }^{20}$ M. Noguchi, K. Hirakawa, and T. Ikoma, Phys. Rev. Lett. 66, 2243 (1991).
${ }^{21}$ R. G. Egdell, S. D. Evans, R. A. Stradling, Y. B. Li, S. D. Parker, and R. H. Williams, Surf. Sci. 262, 444 (1992).

${ }^{22}$ G. A. Baraff and J. A. Appelbaum, Phys. Rev. B 5, 475 (1972).

${ }^{23}$ D. H. Ehlers and D. L. Mills, Phys. Rev. B 34, 3939 (1986).

${ }^{24}$ S. R. Streight and D. L. Mills, Phys. Rev. B 37, 965 (1988).

${ }^{25}$ Y. Chen, S. Nannarone, J. Schaefer, J. C. Hermanson, and G. J. Lapeyre, Phys. Rev. B 39, 7653 (1989).

${ }^{26}$ H. Yu and J. C. Hermanson, Phys. Rev. B 40, 11851 (1989).

${ }^{27}$ T. Inaoka, Surf. Sci. 431, 156 (1999).

${ }^{28}$ G. R. Bell, C. F. McConville, C. P. A. Mulcahy, and T. S. Jones, J. Phys.: Condens. Matter 9, 2903 (1997).

${ }^{29}$ G. R. Bell, T. S. Jones, and C. F. McConvilles, Surf. Sci. 405, 280 (1998).

${ }^{30} \mathrm{Ph}$. Lambin, J. P. Vigneron, and A. A. Lucas, Phys. Rev. B 32, 8203 (1985).

${ }^{31}$ D. H. Ehlers and D. L. Mills, Phys. Rev. B 36, 1051 (1987).

${ }^{32}$ S. R. Streight and D. L. Mills, Phys. Rev. B 40, 10488 (1989).

${ }^{33}$ T. Inaoka, Surf. Sci. 257, 237 (1991).

${ }^{34}$ T. Inaoka, Surf. Sci. 351, 259 (1996).

${ }^{35}$ T. Inaoka, Phys. Rev. B 63, 165322 (2001).

${ }^{36}$ E. O. Kane, J. Phys. Chem. Solids 1, 249 (1957).

${ }^{37}$ M. P. Hasselbeck and P. M. Enders, Phys. Rev. B 57, 9674 (1998).

${ }^{38}$ M. Yamaguchi, T. Inaoka, and M. Hasegawa, Phys. Rev. B 65, 085207 (2002).

${ }^{39}$ T. Inaoka, D. M. Newns, and R. G. Egdell, Surf. Sci. 186, 290 (1987).

${ }^{40}$ J.-P. Zöllner, H. Übensee, G. Paasch, T. Fielder, and G. Gobsch, Phys. Status Solidi B 134, 837 (1986).

${ }^{41}$ J. M. Ziman, Principles of the Theory of Solids (Cambridge University Press, Cambridge, 1972), Chap. 6, Secs. 6.3 and 6.4.

${ }^{42}$ A. L. Fetter and J. D. Walecka, Quantum Theory of ManyParticle Systems (McGraw-Hill, New York, 1971), Chap. 8, Sec. 30 .

${ }^{43}$ R. G. Dandrea, N. W. Ashcroft, and A. E. Carlsson, Phys. Rev. B 34, 2097 (1986).

${ }^{44}$ M. Hass and B. W. Henvis, J. Phys. Chem. Solids 23, 1099 (1962).

${ }^{45}$ F. Lukes, Phys. Status Solidi B 84, K113 (1977).

${ }^{46}$ C. R. Pidgeon, S. H. Groves, and J. Feinleib, Solid State Commun. 5, 677 (1967).

${ }^{47}$ J. Takayama, K. Shimomae, and C. Hamaguchi, Jpn. J. Appl. Phys. 20, 1265 (1981). 